program, in terms of HIV infections averted, far out-weigh the costs from the number of new infections generated from the early resumption of sex see Abstract O2-S2.02 table 1.

Conclusion The resumption of sexual behaviour during the wound healing period poses increased risk for the transmission and acquisition of HIV-1 for individuals. The early resumption of sex is of particular concern for programs that circumcise HIV + men or a large proportion of men who are not HIV tested. However, at the population level, such behaviour seems less of a concern.

Abstract 02-S2.02 Table 1 Sexual behaviour with 6 weeks post MC

\begin{tabular}{ll}
\hline Percent having sex within $\mathbf{6}$ weeks post-MC & \\
\hline Among all MC clients & $24 \%$ \\
Among all MC clients who were sexually active by baseline survey & $30 \%$ \\
Among all MC clients who had sexual partners at baseline & $28 \%$ \\
For those having sex... & $(\mathrm{N}=54)$ \\
Mean number of sex acts in last 4 weeks & 2 (min $=1, \max =12)$ \\
Mean number of partners & $1.6(\min =1, \max =4)$ \\
Reported at least one unprotected sex act & $82 \%$ \\
Reported 2 or more sexual partners in period & $26 \%$ \\
Report sex with a commercial sex worker or bar girl & $4 \%$ \\
Resumed sex within first week of MC & $22 \%$ \\
Resumed sex within first $2^{\text {nd }}$ and 3rd weeks of MC & $24 \%$ \\
Resumed sex $4+$ weeks of MC & $41 \%$ \\
\hline
\end{tabular}

\section{2-S2.03 FEASIBILITY OF GIVING HSV SEROLOGICAL TEST RESULTS BY MAIL AND PHONE}

doi:10.1136/sextrans-2011-050109.75

${ }^{1} \mathrm{M}$ Hayley, ${ }^{1} \mathrm{~T}$ Chikovani, ${ }^{2} \mathrm{~A}$ Rompalo. ${ }^{1} \mathrm{~J}$ ohns Hopkins University School of Nursing, Baltimore, USA; ${ }^{2}$ Johns Hopkins University, School of Medicine, USA

Background Accurate serological tests for the herpes simplex virus have been commercially available since 1999. Despite this availability, they are offered infrequently. Concerns about the resources required for testing including time spent on counselling and followup are among the barriers to testing. The purpose of this study was to determine the feasibility of using letters and phone calls to give HSV-1 and HSV-2 serological test results and to document the resources used to deliver results and respond to subjects' concerns. Methods Subjects were recruited from an urban STD clinic and tested for HSV-1 and HSV-2 as part of a multi-site study of HSV serological test performance $(\mathrm{N}=612)$. Subjects were offered the option of having an investigator mail or call with test results. Result letters included a one page hand-out on HSV-1 and HSV-2 and the phone number of an investigator who could address concerns. Number of phone calls received, time spent on the phone, level of subject distress, and request for referrals were documented. Descriptive statistics were calculated for all variables and data was examined for bivariate relationships using SPSS.

Results Sixty-seven per cent of the subjects requested that their results be mailed $(n=410)$ and $33 \%$ requested that their results be given by phone $(n=202)$. Approximately $6 \%$ of the subjects who received letters called for additional information. Seventy-one per cent of the calls took less than 5 min and only $2 \%$ required more than $10 \mathrm{~min}$. A total of approximately $6.4 \mathrm{~h}$ were spent on the phone to give results and respond to questions. $<1 \%$ of the subjects required multiple phone calls and less than $1 \%$ requested a referral to a clinician. Investigators rated $55 \%$ of subjects spoken to on the phone as not at all distressed, $31 \%$ as somewhat distressed, and $12 \%$ as very distressed. Distress was significantly associated with longer time on the phone, but not with testing positive for HSV-2 vs HSV-1.

Conclusions Delivery of HSV serological test results by phone or mail is feasible and requires minimum time on the part of providers.

\section{2-S2.04 TARGETING HIV PREVENTION EFFORTS ON HIV-INFECTED MEN USING CONDITIONAL CASH TRANSFER (CCT): DOES IT WORK?}

doi:10.1136/sextrans-2011-050109.76

${ }^{1} \mathrm{~A}$ Khan, ${ }^{2} \mathrm{R}$ Oazi, ${ }^{3} \mathrm{~N}$ Nazim, ${ }^{1} \mathrm{~A}$ A Khan. ${ }^{1}$ Research and Development Solutions, Islamabad, Pakistan; ${ }^{2}$ The Pakistan Institute of Medical Sciences Hospital, Pakistan; ${ }^{3}$ The Health Services Academy, Pakistan

Background Preventing HIV infections in female spouses of HIVinfected men is limited by low HIV testing rates among these women who are at an increasingly high risk for HIV acquisition. We explored the effect of conditional cash transfer (CCT) on HIVinfected men for spouse testing and HIV status disclosure to their spouses.

Methods Using medical charts we identified all eligible HIV-infected married men receiving care at the PIMS HIV clinic, Islamabad. Selection criteria for CCT were sexually active married men receiving care $>6$ months including at least two counselling sessions whose spouses had never been tested for HIV (HIV status unknown). CCT was cash given to cover travel/accommodation (US \$14 for out-town and US\$ 5 for in-town) costs for bringing the spouse to the HIV clinic for testing. All study participants underwent a brief study questionnaire looking at patient demographics, visit history, factors influencing spouse testing, barriers to care and self-disclosure. The CCT acceptance rate was $90 \%$ among HIVinfected men. Outcome of interest was spouse testing and status disclosure to spouse at 6 months post CCT.

Results Of the 230 married men, 138 men (60\%) had spouses' never tested/unknown status. Baseline disclosure of HIV status to wife was $29 \%$, and median duration of receiving care was 14.3 months. From these 138 men we were able to contact and enrol 94 (68\%) men for CCT; 53 (56\%) brought their spouses for HIV testing within 4 months; 19 (20\%) self-reported getting their wives tested elsewhere, and only 22 (24\%) did not comply with the CCT conditionality. CCT improved disclosure of HIV status from baseline $29 \%$ to $62 \%(p<0.05)$. Factors associated with spouse testing were men $<50$ years, high ART compliance score, and prior selfdisclosure of status to one family member $(p<0.05)$.

Conclusions Even within the context of a socially conservative society CCT can significantly improve HIV testing rates for female spouses and self-disclosure of HIV status by HIV-infected men. Using CCT for timely prevention of HIV infection in wives and children of HIV positive men reduces risk and can be an effective strategy to overcome socio-cultural and financial barriers. Further studies are needed to explore cost-effectiveness of this approach in preventing new infections.

\section{2-S2.05 START WITH THE SOCIAL DETERMINANTS OF HEALTH TO TAILOR SEXUAL HEALTH PROMOTION FOR FIRST NATIONS, INUIT AND MéTIS YOUTH IN CANADA}

doi:10.1136/sextrans-2011-050109.77

${ }^{1} \mathrm{~J}$ Seto, ${ }^{2} \mathrm{~T}$ Asfaw, ${ }^{3} \mathrm{~K}$ Folz, ${ }^{4} \mathrm{~W}$ Clark, ${ }^{5} \mathrm{M}$ Deleary, ${ }^{1} \mathrm{~A}$ Sheikh. ${ }^{1}$ Public Health Agency of Canada, Ottawa, Canada; ${ }^{2}$ Health Canada, Canada; ${ }^{3}$ Public Health Agency of Canada, Canada; ${ }^{4}$ National Aboriginal Health Organization, Canada; ${ }^{5}$ Assembly of First Nations, Canada

Background Sexual health promotion and approaches for the prevention of sexually transmitted and blood-borne infections (STBBI) should be tailored to the needs and realities of youth. Engaging youth and building their capacity is an important step in health promotion and has proven to be critical in the development of a promising initiative aimed at engaging First Nations, Inuit and Métis youth in Canada. 